\title{
Estratificação da Insuficiência Cardíaca Clínica através do Mapa T1 Nativo: Experiência de um Serviço de Referência
}

\author{
Clinical Heart Failure Stratification Through Native T1 Mapping: Experience of a Referral Service
}

Thiago dos Santos Silva Marques, ${ }^{10}$ André Maurício de Souza Fernandes, ${ }^{2}$ Roberto Nery Dantas Júnior, ${ }^{10}$ Robert W. Biederman, ${ }^{3}$ Ana Paula Marques de Oliveira Melo, ${ }^{4}$ Roque Aras $^{5}{ }^{\mathbb{0}}$

Instituto do Coração do Hospital das Clínicas da Faculdade de Medicina da Universidade de São Paulo, ${ }^{1}$ São Paulo, SP - Brasil

Universidade Federal da Bahia - Faculdade de Medicina de Bahia, ${ }^{2}$ Salvador, BA - Brasil

Allegheny General Hospital, ${ }^{3}$ Pittsburgh, Pennsylvania - EUA

Hospital Heliópolis, ${ }^{4}$ São Paulo, SP - Brasil

Hospital Universitário Professor Edgard Santos, ${ }^{5}$ Salvador, BA - Brasil

\section{Resumo}

Fundamento: Fibrose cardíaca difusa é fator importante na avaliação prognóstica dos pacientes com disfunção ventricular. Mapeamento T1 nativo pela ressonância magnética cardíaca (RMC) apresenta elevada sensibilidade e é considerado preditor independente de mortalidade por todas as causas e desenvolvimento de insuficiência cardíaca (IC) nos pacientes com cardiomiopatia.

Objetivos: Avaliar aplicabilidade da avaliação com mapa T1 nativo em pacientes com IC em um hospital de referência de cardiologia e sua associação com parâmetros estruturais e perfil funcional.

Métodos: Estudo transversal com pacientes adultos com IC classes funcionais NYHA I e II, isquêmicos e não isquêmicos, acompanhados em hospital de referência, que realizaram RMC. Os valores de T1 nativo foram analisados em relação a parâmetros estruturais, comorbidades, etiologia e categorização da IC pela fração de ejeção do ventrículo esquerdo (FEVE). Análises foram realizadas com nível de significância de $5 \%$.

Resultados: Analisados 134 pacientes. Valores de T1 nativo elevados foram encontrados em pacientes com maior dilatação $(1004,9$ vs $1042,7 \mathrm{~ms}, p=0,001)$, volume $(1021,3$ vs $\mathbf{1 0 5 0 , 3} \mathrm{ms}, p<0,01)$ e disfunção ventricular $(1010,1$ vs $1053,4 \mathrm{~ms}, \mathrm{p}<\mathbf{0 , 0 0 1}$ ), mesmo quando analisados isoladamente os não isquêmicos. Pacientes classificados com IC com fração de ejeção reduzida apresentaram maiores valores $\mathrm{T} 1 \mathrm{em}$ relação aos com IC e fração de ejeção preservada (ICFEP) $(992,7$ vs 1054,1 ms, p<0,001). Dos com ICFEP, 55,2\% apresentavam T1 elevado.

Conclusões: Mapeamento T1 por RMC é factível para avaliação da IC clínica. Houve associação direta entre maior valor nativo de T1 e menor fração de ejeção, maiores diâmetros e volumes do VE, independentemente da etiologia da IC. (Arq Bras Cardiol. 2021; 116(5):919-925)

Palavras-chave: Insuficiência Cardíaca; Cardiomiopatia Dilatada; Disfunção Ventricular Esquerda; Fibrose; Diagnóstico por Imagem; Cardiomiopatia Chagásica; Espectroscopia de Ressonância Magnética/métodos.

\footnotetext{
Abstract

Background: Diffuse cardiac fibrosis is an important factor in the prognostic assessment of patients with ventricular dysfunction. Cardiovascular magnetic resonance imaging (CMR) native T1 mapping is highly sensitive and considered an independent predictor of all-cause mortality and heart failure (HF) development in patients with cardiomyopathy.
}

Objectives: To evaluate the feasibility of native T1 mapping assessment in patients with HF in a cardiology referral hospital and its association with structural parameters and functional profile.

Methods: Cross-sectional study with adult patients with HF NYHA functional classes I and II, ischemic and non-ischemic, followed in a referral hospital, who underwent CMR. Native T1 values were analyzed for structural parameters, comorbidities, etiology, and categorization of HF by left ventricular ejection fraction (LVEF). Analyses were performed with a significance level of 5\%.

Correspondência: Thiago dos Santos Silva Marques •

Rua Leonor Calmon, 74, Ed. Principe de Lyon, Apt 1202. CEP 40296-210, Cidade Jardim, Salvador, BA - Brasil

E-mail: thiago.ssm@hotmail.com

Artigo recebido em 12/05/2019, revisado em 08/03/2020, aceito em 15/04/2020

DOI: https://doi.org/10.36660/abc.20190782 
Results: Enrollment of 134 patients. Elevated native T1 values were found in patients with greater dilation (1004.9 vs 1042.7ms, p = 0.001), ventricular volumes (1021.3 vs 1050.3ms, $p<0.01$ ) and ventricular dysfunction (1010.1 vs $1053.4 \mathrm{~ms}, p<0.001$ ), also present when the nonischemic group was analyzed separately. Patients classified as HF with reduced ejection fraction had higher T1 values than those with HF and preserved ejection fraction (HFPEF) (992.7 vs 1054.1ms, p <0.001). Of those with HFPEF, 55.2\% had higher T1.

Conclusions: CMR T1 mapping is feasible for clinical HF evaluation. There was a direct association between higher native T1 values and lower ejection fraction, and with larger LV diameters and volumes, regardless of the etiology of HF. (Arq Bras Cardiol. 2021; 116(5):919-925)

Keywords: Heart Failure; Cardiomyopathy, Dilated; Ventricular Dysfunction, Left; Fibrosis; Diagnosis Imaging; Chagas Cardiomyopathy; Magnetic Resonance Spectroscopy/methods.

Full texts in English - http://www.arquivosonline.com.br

\section{Introdução}

Fibrose cardíaca tornou-se fator importante na avaliação prognóstica dos pacientes com disfunção ventricular, sendo considerada como uma das consequências do remodelamento patológico do ventrículo esquerdo (VE), ${ }^{1}$ que desempenha um marcante papel na resposta do miocárdio à lesão. Quando em excesso leva à progressão da insuficiência cardíaca (IC) e pior prognóstico. ${ }^{2}$ Métodos de imagem não invasivos para avaliação quantitativa, em um estágio inicial, da presença e extensão da fibrose miocárdica são necessários para melhor estratificação de risco de IC e para monitorar os efeitos do tratamento. ${ }^{3}$

Ressonância magnética cardíaca (RMC), considerada ferramenta eficaz para avaliar a morfologia e função miocárdica, bem como alterações teciduais, ${ }^{4-7}$ surgiu como uma modalidade não invasiva de primeira linha para investigação de etiologia e prognóstico em pacientes com disfunção miocárdica. ${ }^{8,9} \mathrm{O}$ mapeamento T1 nativo é um método rápido e sem contraste que visa detectar alterações miocárdicas difusas, em uma variedade de condições cardíacas. Apresenta ampla sensibilidade comprovada para alterações patológicas, incluindo detecção de edema miocárdico, infarto, isquemia, cardiomiopatias e fibrose difusa. ${ }^{10-14}$ Portanto, o mapeamento T1 nativo é um método de imagem alternativo para avaliação da área cardíaca de risco. ${ }^{15}$

Estudo observacional multicêntrico evidenciou que o T1 nativo mostrou ser melhor preditor expressivo de piores desfechos na cardiomiopatia dilatada (CMD) do que os parâmetros clínicos clássicos, mostrando que T1 nativo foi o mais forte preditor independente de mortalidade por todas as causas e desenvolvimento de IC. ${ }^{16,17}$ A gravidade de doença difusa, avaliada pelo mapa T1 nativo, pode ser fisiopatologicamente um parâmetro mais relevante, pois está diretamente relacionada à progressão da doença e à capacidade funcional do miocárdio remanescente. A natureza contínua dos valores de T1 corresponde bem à taxa de eventos clínicos: quanto maior o T1 nativo, maior o risco de eventos adversos e vice-versa. Esses achados permitem refinar a abordagem atual de estratificação de risco em pacientes com cardiomiopatias, principalmente na CMD. ${ }^{17}$

Nosso estudo objetiva avaliar a aplicabilidade da avaliação com mapa T1 nativo em pacientes com IC em um hospital de referência de cardiologia e sua associação com parâmetros estruturais e perfil funcional destes pacientes.

\section{Métodos}

\section{Estudo e Amostra}

Foram incluídos pacientes acompanhados no ambulatório de IC do Hospital Ana Nery, Salvador, Bahia, que consecutivamente foram encaminhados para realização de RMC como parte do cuidado clínico e diagnóstico no período compreendido entre os anos de 2012 e 2016.

Foram selecionados, de forma consecutiva, pacientes com idade $\geq 18$ anos com diagnóstico de IC, de acordo com critérios de Framingham e/ou Boston, conforme Diretriz Brasileira de Insuficiência Cardíaca Crônica e Aguda, com classes funcionais I e II pela New York Heart Association (NYHA), com ao menos IC diastólica tipo II definida pelo ecocardiograma transtorácico, de diversas etiologias, divididas em isquêmicas ou não isquêmicas, definição essa baseada na documentação de infarto do miocárdio (IM) prévio, pela presença de realce tardio pelo gadolínio (RTG) subendocárdico ou transmural (respeitando território coronariano) na RMC e/ou presença de isquemia por algum método diagnóstico. Em relação à cardiomiopatia chagásica, foi considerado o diagnóstico na presença de sorologia positiva e após exclusão de isquemia.

Todos os pacientes realizavam exames de rotina no ambulatório de IC, como radiografia de tórax, teste de caminhada e eletrocardiograma, associado a avaliação de uma equipe multiprofissional. Todos pacientes eram acompanhados no serviço de IC da unidade e faziam uso de terapia medicamentosa otimizada, associada ou não à reabilitação cardíaca pela equipe multidisciplinar, conforme critério clínico do médico assistente.

O trabalho foi aprovado pelo Comitê de Ética e Pesquisa da instituição, como subprojeto do trabalho principal: "Características dos pacientes submetidos à ressonância magnética cardiovascular em um hospital de referência".

\section{Protocolo de Aquisição e Avaliação de Exames de RMC}

Todos os exames de RMC foram realizados em um scanner de corpo inteiro Avanto de 1,5 T (Siemens Medical Solutions, Alemanha) usando uma bobina cardíaca de 8 canais. Imagens em varredura foram realizadas para obter sequências cine SSFP em duas, três e quatro câmaras, além do eixo curto. As imagens de cine foram adquiridas durante apneia expiratória (20 quadros por ciclo cardíaco com cortes de $8 \mathrm{~mm}$ de espessura, FOV 300, matriz $208 \AA$ Åx 80, BW 925 KHz/pixel). Para análise da função do VE, o eixo curto foi composto de 


\section{Artigo Original}

um mínimo de 8 e um máximo de 12 cortes, com 8 mm de espessura e $2 \mathrm{~mm}$ de intervalo.

Imagens de mapa T1 nativo foram realizadas sem injeção de contraste através de corte em porção média do VE através da sequência Modified Look-Locker Inversion recovery (MOLLI), com acoplamento eletrocardiográfico, 250 a 360 mm de FOV; $192 \times 122$ a $192 \times 183$ de tamanho da matriz. Espessura do corte de 6-8 mm; 2,2 / 1,1 ms $\approx \mathrm{TR} / \mathrm{TE}$, ângulo de viragem de $35^{\circ}$; Fator GRAPPA $=2 ; 17$ batimentos cardíacos (que coletam $3+3+5$ amostras). Como a utilização de contraste nos estudos não fazia parte do protocolo e esteve reservado a critério clínico, não foi realizado o cálculo de volume extracelular (VEC) e mapa T1 pós contraste.

O valor de normalidade do T1 nativo, para nossa amostra, foi obtido previamente através de um estudo piloto com pacientes sem comorbidades e coração estruturalmente normal da mesma instituição/scanner, como recomendado pela Society for Cardiovascular Magnetic Resonance (SCMR). ${ }^{18}$ Segundo resultado dessa avaliação, o valor médio de normalidade considerado para o T1 miocárdico nativo foi de $983,46 \pm 34,38 \mathrm{~ms}$.

Todos os exames foram analisados através do software cvi42 (Circle Cardiovascular Imaging Inc., Calgary, Canada) por especialista em imagem cardiovascular com mais de 5 anos de experiência. Após delineamento de bordos endocárdicos e epicárdicos no eixo curto do VE, em sístole e diástole máximas, foram quantificadas variáveis funcionais como fração de ejeção do ventrículo esquerdo (FEVE), diâmetro diastólico final do ventrículo esquerdo (DDFVE), volume diastólico final do ventrículo esquerdo (VDFVE), massa cardíaca (indexados para superfície corpórea), avaliados conforme valores de referência em RMC recomendados. ${ }^{19}$ Para cálculo do mapa T1 nativo, os bordos dos traçados foram realizados de forma estreita com intuito de evitar ao máximo contaminação com a cavidade ventricular ou com gordura epicárdica, e de forma a evitar áreas com realce tardio miocárdico identificável visivelmente (Figura 1). Os exames foram analisados por um único profissional experiente.

Os valores de T1 miocárdico nativo obtidos foram analisados em relação às comorbidades clínicas, parâmetros estruturais, etiologia e categorização da IC. A IC foi categorizada em: 1) FEVE reduzida (IC de fração de ejeção reduzida - ICFER: < 40\%); 2) FEVE limítrofe (IC de fração de ejeção limítrofe - ICFEL: 40 a 49\%); e 3) FEVE preservada (IC de fração de ejeção preservada - ICFEP: $\geq 50 \%)(20,21)$.

\section{Análise Estatística}

Os dados coletados foram descritos através de médias e desvio padrão para as variáveis contínuas de distribuição normal; e medianas e intervalo interquartílico para as de distribuição não normal. As variáveis categóricas foram descritas em números absolutos e porcentagens. A normalidade das variáveis foi testada através do teste de Kolmogorov-Smirnov. Foram realizados testes estatísticos, conforme o tipo de variável e a normalidade da distribuição: teste $t$ de Student não pareado, teste de Mann Whitney e teste do qui-quadrado. Valores de $\mathrm{p}$ inferiores a 0,05 foram considerados estatisticamente significativos. A análise estatística foi realizada utilizando o software SSPS (versão 22.0).

\section{Resultados}

Foram incluídos 134 pacientes no período de janeiro de 2014 a dezembro de 2016. Houve uma predominância de pacientes do sexo masculino, FEVE reduzida e diâmetros/ volumes cavitários aumentados (Tabela 1). Pacientes não isquêmicos foram maioria, num total de 95 indivíduos (70,9\%). Houve presença de realce tardio em 56 pacientes dos 95 com miocardiopatia não isquêmica (59\%), com predomínio

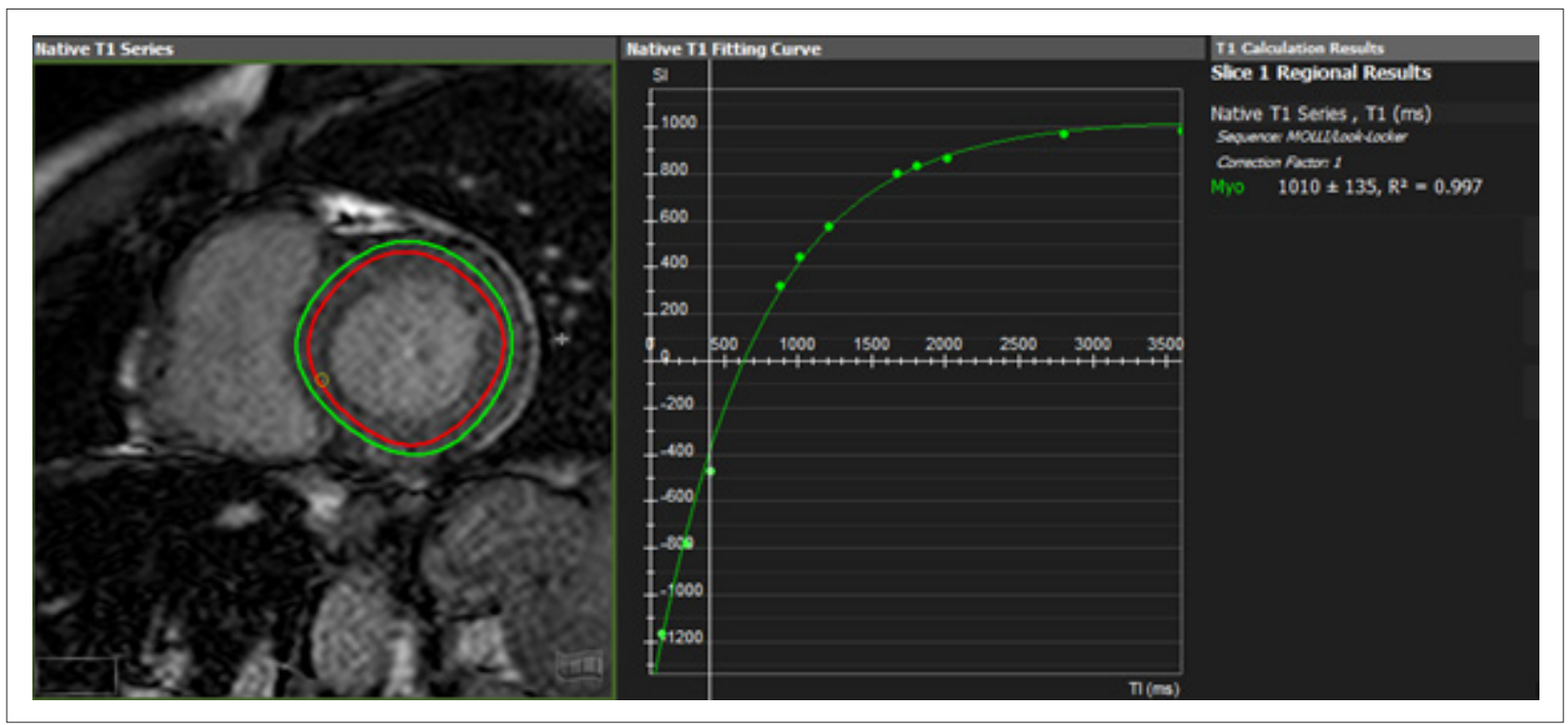

Figura 1 - Cálculo do mapa T1 nativo. Fonte: Marques, 2019. 


\begin{tabular}{lc}
\hline \multicolumn{2}{l}{ Tabela 1 - Características clínicas e funcionais da amostra } \\
\hline Características gerais & $\mathbf{n}(134)$ \\
\hline Idade (anos) (DP) & $50,2(14,0)$ \\
\hline Sexo masculino (\%) & $94(70,1 \%)$ \\
\hline Etiologia não-isquêmica & $95(70,9 \%)$ \\
\hline Átrio esquerdo (cm) (DP) & $3,9(0,8)$ \\
\hline Septo interventricular (cm) (DP) & $0,8(0,2)$ \\
\hline Parede posterior (cm) (DP) & $0,7(0,2)$ \\
\hline FEVD (\%) (DP) & $39,6(15,9)$ \\
\hline FEVE (\%) (DP) & $34,4(17,9)$ \\
\hline DDFVE (cm) (DP) & $6,4(1,2)$ \\
\hline DSFVE (cm) (DP) & $5,1(1,6)$ \\
\hline VDFVE (ml) (DP) & $215,1(96,2)$ \\
\hline VDFVE indexado (ml/m²) (DP) & $116,7(51,9)$ \\
\hline VSFVE (ml) (DP) & $150,9(93,7)$ \\
\hline VSFVE indexado (ml/m²) (DP) & $82,5(52,3)$ \\
\hline MC (g) (IQ) & $88,5(73,7 ; 114,0)$ \\
\hline MC indexada (g/m²) (IQ) & $49,0(40,0 ; 62,5)$ \\
\hline Hipertensão arterial & $53(39,6 \%)$ \\
\hline Diabetes & $21(15,7 \%)$ \\
\hline Doença coronária & $33(24,6 \%)$ \\
\hline Doença renal crônica & $13(9,7 \%)$ \\
\hline Tabagismo & $20(14,9 \%$ \\
\hline Chagas & $13(9,7 \%)$ \\
\hline Dislipidemia & $7,2 \%)$ \\
\hline FEVE: Fran \\
\hline
\end{tabular}

FEVE: Fração de ejeção do ventrículo esquerdo; FEVD: Fração de ejeção do ventrículo direito; DDFVE: Diâmetro diastólico final do ventrículo esquerdo; DSFVE: Diâmetro sistólico final do ventrículo esquerdo; VDFVE: Volume diastólico final do ventrículo esquerdo; VSFVE: Volume sistólico final do ventrículo esquerdo; MC: Massa cardiaca; DP: Desvio padrão; IQ: Intervalo interquartilico. Fonte: Marques, 2019. Não esta nas referências

de realce mesocárdico e multifocal. Dentre os pacientes com miocardiopatia isquêmica, 34 pacientes (87\%) apresentaram realce tardio, em sua maioria transmural.

Valores de T1 miocárdico nativo elevados, quando analisado em relação ao VE, foram encontrados em pacientes com maior dilatação $(p=0,007)$, maiores volumes ventriculares $(p<0,01)$ e disfunção ventricular $(p<0,001)$ (Tabela 2$)$. Numa avaliação adicional de forma dicotomizada, considerando-se essas mesmas variáveis funcionais, manteve-se associação do valor do T1 miocárdico nativo, conforme exposto na Tabela 3. Quando realizada a análise de subgrupo dos pacientes não isquêmicos, as mesmas associações encontradas permaneceram presentes (Tabelas 3 e 4). Houve adequada concordância intraobservador na detecção de valores T1 elevados (Kappa 0,82; $p=0,001$ ).
Quando analisado o T1 miocárdico nativo em relação ao perfil da IC, classificada conforme a FEVE, observou-se um maior valor T1 nos pacientes com FEVE $<35 \%$ ( $p<0,001)$ (Tabela 5). Houve diferença significativa entre os grupos, com T1 mais elevado, quando comparado ICFER com ICFEL $(p=0,004)$; e com ICFEP $(p<0,001)$; assim como quando comparado ICFEL com ICFEP $(p=0,02)$. Dos pacientes com ICFEP, 55,2\% já apresentavam T1 elevado. Quando analisado em relação aos diâmetros e volumes cavitários, observados maiores valores nos pacientes com ICFER e ICFEL quando comparados com os ICFEP $(p<0,01)$.

Quanto à etiologia da IC, foi observado que, independente da etiologia, há alta porcentagem de pacientes com T1 nativo elevado ( $89,7 \%$ nos isquêmicos e $81,1 \%$ nos não isquêmicos), com um maior valor T1 nos isquêmicos em relação aos não isquêmicos $(p=0,004)$. Analisando especificamente o grupo de não isquêmicos, 13 pacientes tinham diagnóstico de cardiomiopatia chagásica, todos apresentam T1 nativo elevado $(1077,1 \pm 61,1 \mathrm{~ms})$ associado a FEVE reduzida $(27,6$ $\pm 16,8 \%$, elevados DDFVE $(7,1 \pm 1,5 \mathrm{~cm})$, DSFVE $(6,1 \pm$ $1,7 \mathrm{~cm})$, VDFVE indexado $\left(146,7 \pm 52,3 \mathrm{ml} / \mathrm{m}^{2}\right)$ e VSFVE indexado $\left(112,7 \pm 54,1 \mathrm{ml} / \mathrm{m}^{2}\right)$

Dentre as comorbidades clínicas avaliadas, observa-se associação estatística de maiores valores de T1, acima da faixa de normalidade, nos pacientes tabagistas $(p=0,032)$. (Tabela 6)

\section{Discussão}

O presente estudo demonstra que, além de o Mapeamento T1 ser factível na prática clínica, existe associação do T1 miocárdico nativo com disfunção miocárdica, expressa por uma menor FEVE e maiores volumes e diâmetros intracavitários, independentemente da etiologia da cardiomiopatia.

A RMC permite a detecção de fibrose miocárdica difusa através do mapeamento T1 nativo e com alta concordância com a biópsia miocárdica. ${ }^{6} \mathrm{Um}$ estudo recente publicado com 637 pacientes com CMD não isquêmica demonstrou que a presença de fibrose por mapeamento T1 nativo está relacionada ao desfecho combinado de mortalidade por todas as causas de IC ( $p<0,001)$, e na análise multivariada, é considerado como um preditor independente para esses desfechos (IC 1,06-1,15, p <0,001). ${ }^{16}$ Estudo prévio validou o uso do mapeamento T1 para confirmação de fibrose com excelente correlação $(R=0,95, p<0,001)$ entre o exame de RMC e a histologia, mas quando analisado comparando com o RTG, este último foi menos acurado na avaliação da fibrose intersticial difusa. ${ }^{6}$ Assim, o mapeamento T1 nativo do miocárdio é um método de imagem que permite detectar a presença de fibrose com maior precocidade que o RTG, que pode ser relacionada a um pior prognóstico. ${ }^{22}$

Entre as etiologias, no Brasil, há uma característica distinta em relação à prevalência e importância da doença de Chagas. ${ }^{23,24}$ No presente estudo, houve uma prevalência de 9,7\% de cardiomiopatia Chagásica, que representa 13,7\% dos pacientes não isquêmicos. Todos pacientes chagásicos tiveram valores nativos de T1 elevados, sendo observado um maior T1 nativo associado com menor FEVE, maior DDVE e VDFVE quando comparado com os demais não isquêmicos com T1 elevado, mas sem significância estatística. Estudos anteriores 


\section{Artigo Original}

\begin{tabular}{lccc}
\hline Tabela 2 - Avaliação do mapa T1 miocárdico nativo com parâmetros funcionais & & & \\
\hline & T1 normal (ms) & T1 elevado (ms) & p \\
\hline FEVE (\%) (DP) & $50,27(16,3)$ & $31,26(16,5)$ & $<0,001^{*}$ \\
\hline DDFVE (cm) (DP) & $5,74(1,2)$ & $6,55(1,2)$ & $0,007^{*}$ \\
\hline DSFVE (cm) (DP) & $3,95(1,42)$ & $5,32(1,5)$ & $<0,001^{*}$ \\
\hline VDFVE (ml) (IQ) & $167,9(62,5)$ & $224,3(99,1)$ & $0,001^{*}$ \\
\hline VDFVE indexado (ml/m²) (DP) & $89,8(31,1)$ & $122,0(53,7)$ & $0,001^{*}$ \\
\hline VSFVE (ml) (DP) & $91,5(60,2)$ & $162,6(94,8)$ & $0,001^{*}$ \\
\hline VSFVE indexado (ml/m²) (DP) & $47,9(30,9)$ & $89,3(53,1)$ & $0,001^{*}$ \\
\hline MaSsa (g) (IQ) & $81,0(66,0 ; 99,2)$ & $89,5(77,0 ; 119,5)$ & $0,05^{\dagger}$ \\
\hline Massa indexada $\left(\mathrm{g} / \mathrm{m}^{2}\right)(I Q)$ & $41,5(36,5 ; 52,5)$ & $50,0(40,5 ; 62,7)$ & $0,025^{\dagger}$ \\
\hline
\end{tabular}

FEVE: Fração de ejeção do ventrículo esquerdo; DDFVE: Diâmetro diastólico final do ventrículo esquerdo; DSFVE: Diâmetro sistólico final do ventrículo esquerdo; VDFVE: Volume diastólico final do ventrículo esquerdo; VSFVE: Volume sistólico final do ventrículo esquerdo; MC: massa cardíaca; DP: desvio padrão; IQ: intervalo interquartilico. * Teste T de student. ${ }^{\dagger}$ Teste de Mann-Whitney Fonte: Marques, 2019. Não está nas referências

Tabela 3 - Avaliação do valor T1 miocárdico nativo com parâmetros funcionais na amostra geral e não isquêmicos

\begin{tabular}{|c|c|c|c|c|c|}
\hline & & \multicolumn{2}{|c|}{ Geral } & \multicolumn{2}{|c|}{ Não Isquêmicos } \\
\hline & & $\mathrm{T} 1$ (ms) & $\mathrm{p}$ & $\mathrm{T} 1$ (ms) & $\mathrm{p}$ \\
\hline \multirow{2}{*}{ FEVE (\%) (DP) } & $>35 \%$ & $1010,1(46,6)$ & \multirow{2}{*}{$<0,001$} & $1008,9(43,7)$ & \multirow{2}{*}{$<0,001$} \\
\hline & $<35 \%$ & $1053,4(48,1)$ & & $1052,1(48,1)$ & \\
\hline \multirow{2}{*}{ DDFVE (cm) (DP) } & Normal & $1004,9(48,1)$ & \multirow{2}{*}{0,001} & $1010,8(39,9)$ & \multirow{2}{*}{0,03} \\
\hline & Dilatado & $1042,7(50,4)$ & & $1038,3(53,4)$ & \\
\hline \multirow{2}{*}{ DSFVE (cm) (DP) } & Normal & $989,0(43,7)$ & \multirow{2}{*}{$<0,001$} & $994,2(37,7)$ & \multirow{2}{*}{0,001} \\
\hline & Dilatado & $1043,8(49,0)$ & & $1040,3(51,1)$ & \\
\hline \multirow{2}{*}{ VDFVE indexado $\left(\mathrm{ml} / \mathrm{m}^{2}\right)(\mathrm{DP})$} & Normal & $1021,3(49,3)$ & \multirow{2}{*}{0,001} & $1015,5(46,0)$ & \multirow{2}{*}{0,001} \\
\hline & Aumentado & $1050,4(50,8)$ & & $1049,2(52,4)$ & \\
\hline \multirow{2}{*}{ VSFVE indexado $\left(\mathrm{ml} / \mathrm{m}^{2}\right)(\mathrm{DP})$} & Normal & $1000,7(48,3)$ & \multirow{2}{*}{$<0,001$} & $999,8(42,5)$ & \multirow{2}{*}{$<0,001$} \\
\hline & Aumentado & $1048,5(47,3)$ & & $1046,2(49,5)$ & \\
\hline
\end{tabular}

FEVE: Fração de ejeção do ventrículo esquerdo; DDFVE: Diâmetro diastólico final do ventrículo esquerdo; DSFVE: Diâmetro sistólico final do ventrículo esquerdo; VDFVE: Volume diastólico final do ventrículo esquerdo; VSFVE: Volume sistólico final do ventrículo esquerdo; DP: desvio padrão. Teste T de student. Fonte: Marques, 2019. Não está nas referências

menores evidenciaram uma associação estatisticamente significativa $(p<0,001)$ entre a presença de fibrose com piores desfechos nesses pacientes, principalmente relacionada a eventos arrítmicos. ${ }^{23,24} \mathrm{Em}$ estudo anterior foi demonstrado que a probabilidade de taquicardia ventricular (TV) foi maior na presença de fibrose transmural por realce tardio, sendo um preditor de TV clínica (RR 4,1, $p=0,04){ }^{23}$

Há limitações que devem ser destacadas principalmente relacionadas ao modelo transversal do estudo. O tamanho da amostra foi limitado, o que impede a validação adequada das conclusões encontradas. Algumas patologias adicionais podem levar a alterações do T1, incluindo fibrose miocárdica difusa por outras causas, edema, inflamação e doenças infiltrativas. Como não foi realizado estudo de mapa T1 pós-contraste, o cálculo e avaliação do VEC não foi possível, o que não reduz a importância dos achados, uma vez que o T1 nativo demonstrou na literatura ser comparável ao VEC na quantificação de colágeno demonstrado histologicamente. ${ }^{25}$ Apesar de ter sido realizado e analisado de acordo com recomendações prévias, como o mapeamento T1 é um método relativamente novo, ainda requer padronização metodológica. ${ }^{26}$

\section{Conclusões}

A utilização do mapa T1 miocárdico nativo é factível para avaliação da IC clínica, com correlação significativa a um pior perfil funcional. Houve associação direta entre maior valor nativo de T1 e piores parâmetros clínicos e funcionais, 


\begin{tabular}{|c|c|c|c|}
\hline & T1 normal (ms) & T1 elevado (ms) & $\mathbf{p}$ \\
\hline FEVE (\%) (DP) & $48,9(16,6)$ & $32,3(17,9)$ & $0,001^{*}$ \\
\hline DDFVE (cm) (DP) & $5,9(1,2)$ & $6,6(1,4)$ & $0,035^{*}$ \\
\hline DSFVE $(\mathrm{cm})(\mathrm{DP})$ & $4,0(1,5)$ & $5,4(1,7)$ & $0,002^{*}$ \\
\hline $\operatorname{VDFVE~(ml)~(IQ)~}$ & $173,7(66,8)$ & $236,5(112,8)$ & $0,003^{*}$ \\
\hline VDFVE indexado $\left(\mathrm{ml} / \mathrm{m}^{2}\right)$ (DP) & $92,2(30,6)$ & $122,7(60,9)$ & $0,001^{*}$ \\
\hline VSFVE (ml) (DP) & $97,7(63,3)$ & $170,5(107,9)$ & $<0,001^{*}$ \\
\hline VSFVE indexado $\left(\mathrm{ml} / \mathrm{m}^{2}\right)(\mathrm{DP})$ & $50,1(31,2)$ & $93,0(60,2)$ & $<0,001^{*}$ \\
\hline Massa (g) (IQ) & $84,5(66,7 ; 99,2)$ & $91,0(77,0 ; 129,0)$ & $0,06^{\dagger}$ \\
\hline Massa indexada $\left(\mathrm{g} / \mathrm{m}^{2}\right)(\mathrm{IQ})$ & $42,0(37,7 ; 52,5)$ & $56,2(42,0 ; 95,0)$ & $0,02^{\dagger}$ \\
\hline
\end{tabular}

FEVE: Fração de ejeção do ventrículo esquerdo; DDFVE: Diâmetro diastólico final do ventrículo esquerdo; DSFVE: Diâmetro sistólico final do ventrículo esquerdo; VDFVE: Volume diastólico final do ventrículo esquerdo; VSFVE: Volume sistólico final do ventrículo esquerdo; MC: massa cardíaca; DP: desvio padrão; IQ: intervalo interquartilico. ${ }^{*}$ Teste T de student. ${ }^{\dagger}$ Teste de Mann-Whitney. Fonte: Marques, 2019. Não está nas referências

Tabela 5 - Avaliação do mapa T1 miocárdico nativo com classificação de IC

\begin{tabular}{llccc}
\hline & N & T1 normal (ms) & T1 elevado (ms) & p \\
\hline ICFER & 84 & $5(6 \%)$ & $79(94 \%)$ & $<0,001^{*}$ \\
\hline ICFEL & 21 & $4(19 \%)$ & $17(81 \%)$ & $16(55 \%)$ \\
\hline ICFEP & 29 & $13(45 \%)$ & & \\
\hline
\end{tabular}

ICFER: Insuficiência cardíaca com fração de ejeção reduzida; ICFEL: Insuficiência cardíaca com fração de ejeção limitrofe; ICFEP: Insuficiência cardiaca com fração de ejeção preservada. *Teste de qui-quadrado. Fonte: Marques, 2019.Não está nas referências

Tabela 6 - Avaliação do mapa T1 miocárdico nativo com comorbidades clínicas

\begin{tabular}{|c|c|c|c|}
\hline & T1 normal & T1 elevado & $\mathbf{p}$ \\
\hline Hipertensão arterial (\%) & $9(17,0 \%)$ & $44(83,0 \%)$ & 0,88 \\
\hline Diabetes (\%) & $1(4,8 \%)$ & $20(95,2 \%)$ & 0,11 \\
\hline Doença coronária (\%) & $4(12,1 \%)$ & $29(87,9 \%)$ & 0,44 \\
\hline Doença renal crônica (\%) & $0(0 \%)$ & $13(100 \%)$ & 0,09 \\
\hline Tabagismo (\%) & $0(0 \%)$ & $20(100 \%)$ & 0,03 \\
\hline Chagas (\%) & $1(7,1 \%)$ & $13(92,9 \%)$ & 0,09 \\
\hline Dislipidemia (\%) & $1(14,3 \%)$ & $6(85,7 \%)$ & 0,87 \\
\hline
\end{tabular}

Teste de qui-quadrado. Fonte: Marques, 2019. Não está nas referências

dentre eles uma menor fração de ejeção, diâmetros e volumes maiores do VE, independentemente da etiologia da cardiomiopatia. É importante ressaltar que, em pacientes com cardiopatia chagásica, patologia prevalente no Brasil, a mesma associação foi observada.

\section{Agradecimentos}

Agradeço aos Prof. Dr. Roque Aras, Prof. Dr. André Maurício Fernandes, Dr. Roberto Nery e Dr. Robert Biederman pelo apoio, orientação e revisão do projeto e texto final do artigo. Agradeço a Ana Paula Marques pela ajuda na análise dos dados e construção da base de dados.

\section{Contribuição dos Autores}

Concepção e desenho da pesquisa: Marques TSS, Fernandes AMS, Dantas Júnior RN, Aras R; Obtenção de dados, Análise e interpretação dos dados e Análise estatística: Marques TSS, Melo APMO; Redação do manuscrito: Marques TSS; Revisão crítica do manuscrito quanto ao conteúdo 
intelectual importante: Fernandes AMS, Dantas Júnior RN, Biederman RW, Aras R.

\section{Potencial Conflito de Interesses}

Declaro não haver conflito de interesses pertinentes.

\section{Fontes de Financiamento}

O presente estudo não teve fontes de financiamento externas.

\section{Referências}

1. Mann DL, Barger PM, Burkhoff D. Myocardial Recovery and the Failing Heart. J Am Coll Cardiol. 2012;60(24):2465-72.

2. Brown RD, Ambler SK, Mitchell MD, Long CS. The Cardiac fibrolast: Therapeutic Target in Myocardial Remodeling and Failure. Annu Rev Pharmacol Toxicol. 2005;45(1):657-87.

3. Chen R, Abendschein D, Goldstein T, Yin Q, Muccigrosso D, O'Connor R, et al. A non-contrast CMR index for assessing myocardial fibrosis. Magn Reson Imaging . 2017;42:69-73.

4. Vitorino RR, Nacif MS. Ressonância magnética cardíaca na cardiomiopatia dilatada : atualidades. Rev Bras Clin Med. 2011;9(3):225-33.

5. Hombach V, Merkle N, Torzewski J, Kraus JM, Kunze M, Zimmermann $\mathrm{O}$, et al. Electrocardiographic and cardiac magnetic resonance imaging parameters as predictors of a worse outcome in patients with idiopathic dilated cardiomyopathy. Eur Heart J. 2009;30(16):2011-8.

6. Iles LM, Ellims AH, Llewellyn H, Hare JL, Kaye DM, McLean CA, et al. Histological validation of cardiac magnetic resonance analysis of regional and diffuse interstitial myocardial fibrosis. Eur Heart J Cardiovasc Imaging. $2015 ; 16(1): 14-22$

7. Abdel-Aty H, Friedrich MG. Magnetic resonance of cardiomyopathies and myocarditis. In: Kwong RY, (editor). Cardiovascular magnetic resonance imaging. New Jersey: Humana Press; 2008. p:399-414.

8. Stirrat J, White JA, Wisenberg G, Drangova M, Gula L, Skanes A, et al. Prediction of Arrhythmic Events in Ischemic and Dilated Cardiomyopathy Patients Referred for Implantable Cardiac Defibrillator. Circ Cardiovasc Imaging. 2012;5(4):448-56.

9. Yasuda S, Kanzaki H, Ogawa H, Ishibashi-Ueda H, Morita Y, Yamada N, et al. Prognostic impact of blood pressure response plus gadolinium enhancement in dilated cardiomyopathy. Heart. 2015;101(10):774-80.

10. Ferreira VM, Piechnik SK, Robson MD, Neubauer S, Karamitsos TD. Myocardial tissue characterization by magnetic resonance imaging: Novel applications of T1 and T2 mapping. J Thorac Imaging. 2014;29(3):147-54.

11. Moon JC, Messroghli DR, Kellman P, Piechnik SK, Robson MD, Ugander M, et al. Myocardial T1 mapping and extracellular volume quantification: A Society for Cardiovascular Magnetic Resonance (SCMR) and CMR Working Group of the European Society of Cardiology consensus statement. J Cardiovasc Magn Reson. 2013;15(1):1.

12. Piechnik SK, Ferreira VM, Dall'Armellina E, Cochlin LE, Greiser A, Neubauer $\mathrm{S}$, et al. Shortened Modified Look-Locker Inversion recovery (ShMOLLI) for clinical myocardial T1-mapping at 1.5 and $3 \mathrm{~T}$ within a 9 heartbeat breathhold. J Cardiovasc Magn Reson. 2010 Dec 19;12(1):69.

13. Whelan CJ, Ntusi NB, Neubauer S, Karamitsos TD, Myerson SG, Ferreira VM, et al. Noncontrast T1 Mapping for the Diagnosis of Cardiac Amyloidosis. JACC Cardiovasc Imaging. 2013;6(4):488-97.

\section{Vinculação Acadêmica}

Este artigo é parte de dissertação de Mestrado de Thiago dos Santos Silva Marques pela Universidade Federal da Bahia.

\section{Aprovação Ética e Consentimento Informado}

Este estudo foi aprovado pelo Comitê de Ética do Hospital Ana Nery sob o número de protocolo 171.522. Todos os procedimentos envolvidos nesse estudo estão de acordo com a Declaração de Helsinki de 1975, atualizada em 2013.

14. Roujol S. Reproducibility of Four T1 Mapping Sequences : A Head-. Radiology. 2014;272(3):683-9.

15. Muscogiuri G, Suranyi P, Schoepf UJ, De Cecco CN, Secinaro A, Wichmann JL, et al. Cardiac Magnetic Resonance T1-Mapping of the Myocardium: Technical Background and Clinical Relevance. J Thorac Imaging. 2018;33(2):71-80.

16. Puntmann VO, Carr-White G, Jabbour A, Yu CY, Gebker R, Kelle S, et al. T1-Mapping and Outcome in Nonischemic Cardiomyopathy All-Cause Mortality and Heart Failure. JACC Cardiovasc Imaging. 2016;9(1):40-50.

17. Satoh H. Distribution of late gadolinium enhancement in various types of cardiomyopathies: Significance in differential diagnosis, clinical features and prognosis. World J Cardiol. 2014;6(7):585

18. Messroghli DR, Moon JC, Ferreira VM, Grosse-Wortmann L, He T, Kellman $\mathrm{P}$, et al. Clinical recommendations for cardiovascular magnetic resonance mapping of T1, T2, T2 and extracellular volume: A consensus statement by the Society for Cardiovascular Magnetic Resonance (SCMR) endorsed by the European Association for Cardiovascular Imagin. J Cardiovasc Magn Reson. 2017;19(1):1-24.

19. Kawel-boehm N, Maceira A, Valsangiacomo-buechel ER, Vogel-claussen J, Turkbey EB, Williams R, et al. Normal values for cardiovascular magnetic resonance in adults and children. J Cardiovasc Magn Reson. 2015;17(1):29.

20. Fontes-Carvalho R. Insuficiência Cardíaca com Fração de Ejeção Preservada : Combater Equívocos para uma Nova Abordagem. Arq Bras Cardiol. 2011;96(6):504-14

21. Ponikowski P, Voors A. 2016 Esc guidelines for the diagnosis and treatment of acute and chronic heart failure: The Task Force for the diagnosis and treatment of acute and chronic heart failure of the European society of cardiology (ESC): Developed with the special contribution . Russ J Cardiol. 2017;141(1):7-81.

22. Liu JM, Liu A, Leal J, McMillan F, Francis J, Greiser A, et al. Measurement of myocardial native T1 in cardiovascular diseases and norm in 1291 subjects. J Cardiovasc Magn Reson. 2017;19(1):1-10.

23. Espinosa MM, Cirenza C, Szarf G, Szejnfeld D, Mello RP de, Lima JAC, et al. Realce Tardio miocárdico por Ressonância Magnética Cardíaca pode identificar risco para Taquicardia Ventricular na Cardiopatia Chagásica Crônica. Arq Bras Cardiol. 2012;98(5):421-30.

24. Senra T, lanni BM, Costa ACP, Mady C, Martinelli-Filho M, Kalil-Filho R, et al. Long-Term Prognostic Value of Myocardial Fibrosis in Patients With Chagas Cardiomyopathy. J Am Coll Cardiol. 2018;72(21):2577-87.

25. Nakamori S, Dohi K, Ishida M, Goto Y, Imanaka-yoshida K, Omori T, et al. Native T1 Mapping and Extracellular Volume Mapping for the Assessment of Diffuse Myocardial Fibrosis in Dilated\&nbsp;Cardiomyopathy. JACC Cardiovasc Imaging. 2018;11(1):48-59.

26. Fuad Jan M, Jamil TajikA. Modern imaging techniques in cardiomyopathies. Circ Res. 2017;121(7):874-91. 